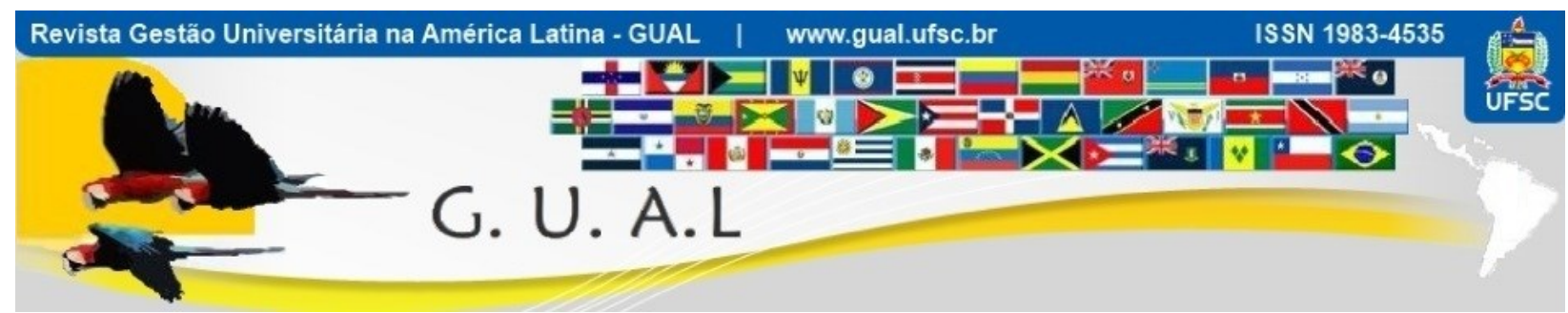

DOI: http://dx.doi.org/10.5007/1983-4535.2015v8n4p117

\title{
SENTIDO DO TRABALHO E ORIENTAÇÃO PARA O TRABALHO: UM ESTUDO EM UNIVERSIDADES PÚBLICAS DE MINAS GERAIS E DO QUEBEC
}

THE MEANING OF WORK AND WORK ORIENTATION: A STUDY IN PUBLIC UNIVERSITIES OF MINAS GERAIS AND QUEBEC

Ana Alice Vilas Boas, Pós-Doutora Universidade Federal de Lavras ana.alice2006@yahoo.com

Estelle M. Morin, Doutora HEC - Montreal estelle.morin@hec.ca

Recebido em 05/dezembro/2014

Aprovado em 05/abril/2015

Sistema de Avaliação: Double Blind Review

Esta obra está sob uma Licença Creative Commons Atribuição-Uso. 


\title{
RESUMO
}

Este estudo avalia a relação entre sentido do trabalho e a orientação para o trabalho de professores universitários de Minas Gerais e do Quebec e correlaciona estes resultados com questões de Qualidade de Vida no Trabalho. A amostra foi constituída por 274 professores de Minas Gerais e 252 professores do Quebec. Os dados foram coletados através de um questionário online enviado, para 3 universidades públicas de Minas Gerais e 3 universidades do Quebec, no primeiro semestre de 2013. Foram encontradas diferenças significativas entre as médias de percepção dos dois grupos de professores para meu trabalho é um emprego e meu trabalho é um carreira. Com relação ao sentido do trabalho e sentido no trabalho observou-se que estes dois indicadores de qualidade de vida no trabalho estão diretamente relacionados com a orientação para a vocação profissional dos professores que participaram da pesquisa. Estes resultados podem orientar os gestores universitários a conduzirem ações que melhorem as condições do ambiente físico de trabalho e as relações de trabalho, pois são estes aspectos que ajudam a melhorar a qualidade de vida no trabalho e a dar mais sentido ao trabalho como um todo.

Palavras-chaves: Vocação. Carreira. Gestão de Pessoas. QVT.

\begin{abstract}
This study evaluates the relationship between the meaning of work and job orientation for professors of Minas Gerais and Quebec and correlate these results with Quality of Working Life. The sample consisted of 274 professors from Minas Gerais and 252 professors from Quebec. Data were collected through an online questionnaire sent to three public universities of Minas Gerais and three universities in Quebec, during the first semester of 2013. There were significant differences between the means of perception of the two groups of professors for my work is a job and my work is a career. Regarding the meaning of work and the meaning at work, it was observed that these two quality of working life indicators are directly related to calling for the professors who participated in the survey. These results can guide university administrators to conduct actions to improve the conditions of physical work environment and job relations, as these aspects may help to improve the quality of working life and give more meaning to the work as a whole.
\end{abstract}

Key-words: Calling. Career. People Management. QWL. 


\section{INTRODUÇÃO}

A carreira acadêmica que já foi vista como segura e como um ambiente de alta posição social, com oportunidades de trabalho satisfatórias e autônomas, atualmente foi alterada drasticamente. O crescimento do número de alunos e o aumento da ênfase na pesquisa, atrelados a pressão econômica que afeta o nível de demanda de trabalho para professores e outros membros da faculdade afetam o ambiente e vida dos profissionais do ensino. Todas essas mudanças afetam negativamente a saúde física e mental no domínio acadêmico.

Gillespie, Walsh, Winefields, Dua e Stough (2001) afirmam que os funcionários das universidades desempenham um papel vital na criação e desenvolvimento do conhecimento e da inovação, bem como na educação e formação de toda a sociedade. Assim, é importante que os gestores encontrem maneiras de proteger os professores e os outros membros da equipe dos níveis crescentes de estresse, devido às exigências cada vez maiores. Como exemplo, podemos destacar a enorme demanda para a publicação em periódicos de alta qualidade para apoiar a carreira do profissional e manter os programas de pós-graduação. Por conseguinte, os professores precisam assumir atividades técnicas, a fim de apresentar projetos de pesquisa, realizar pesquisas e elaborar relatórios para obter material adequado para publicar.

Sobre este assunto, muitos estudos focam o papel "Publicar ou perecer" e esse papel tem aumentado o estresse acadêmico para professores e pesquisadores e tem com isso afetado a qualidade de vida no trabalho. É porque, para publicar os professores precisam trabalhar mais horas e se dedicar às suas próprias pesquisas e as pesquisas de seus alunos de mestrado, doutorado e pós-doutoramento. Todas essas pressões levam o estresse relacionados ao trabalho, desequilíbrio entre vida e trabalho, tensão mental, física e emocional, e pode causar diferentes problemas de saúde e até mesmo de relacionamento no ambiente de trabalho. Em outras palavras, podemos dizer que a QVT para os acadêmicos tem sido drasticamente afetada pelas exigências do trabalho, ou mais precisamente pelas demandas de publicação.

Para ajudar a enfrentar esta realidade, os gestores das universidades devem buscar alternativas para oferecer um ambiente de trabalho saudável e estimulante. O que pode ser entendido como uma melhoria na qualidade de vida dos profissionais. Ketchum e Trist (1992) definiram "qualidade da vida no trabalho" como o estado geral de bem-estar no local de trabalho. Essa qualidade pode ser avaliada por diversos indicadores tais como bem-estar psicológico, sofrimento psicológico, comprometimento organizacional e equilíbrio trabalhovida (Vilas Boas \& Morin, 2013, 2013a, 2013b). E, por fatores como objetivo claro de 
trabalho, autonomia, oportunidades de desenvolvimento profissional, reconhecimento, boas relações com colegas e superiores, percepção de retidão moral no trabalho e nas relações de trabalho, segurança no emprego e carga de trabalho adequada (Vilas Boas \& Morin, 2014). Adicionalmente, torna-se relevante estudar a orientação profissional dos professores universitários para ajudar a direcionar as ações de qualidade de vida nestas instituições.

No entanto, pouco se sabe sobre a orientação profissional nas faculdades ou universidades. Portanto, esse trabalho concentra-se na qualidade de vida no trabalho em universidades públicas no Brasil e no Canadá. Assim, o objetivo é analisar a percepção dos professores universitários sobre a orientação destes profissionais frente ao trabalho acadêmico e determinar as principais diferenças em relação a orientação profissional nesses dois países.

\section{REFERENCIAL TEÓRICO}

\subsection{QUALIDADE DE VIDA NO TRABALHO}

O termo "Qualidade de Vida no Trabalho" foi introduzido pela primeira vez em uma conferência internacional realizada em Arden House em 1972, por Louis Davis. As comunicações que foram apresentadas, em seguida, foram publicadas em dois volumes (Davis \& Cherns, 1975). Esta conferência literalmente lançou todo um campo de pesquisa em desenho do trabalho conhecido como Projeto de Sistemas Sócio Técnico (STSD). QVT é agora um conceito complexo, que combina dois aspectos principais: os fatores - determinantes da QVT e os indicadores - componentes de QVT.

Boisvert (1977) publicou uma análise da qualidade de vida no trabalho com 15 dimensões de QVT: controle ou autonomia no trabalho, capacidade de exercer um juízo, importância das decisões, oportunidades de aprendizagem, uso de habilidades, controle sobre os critérios de desempenho, desafios apresentados no trabalho, variedade de tarefas, interação com os colegas, reconhecimento, orgulho de realização, contribuição do trabalho para os objetivos da organização, percepção de um futuro desejável e participação na tomada de decisões. Esta lista apresenta diferentes dimensões que são ou fatores, como a interação com os colegas, ou indicadores, como o orgulho da realização.

Levine (1983) publicou uma medida desenvolvida através do método de Delphi em uma amostra representativa de 70 empregados. Foram identificados seis critérios com este método: o respeito e a confiança dos gerentes com funcionários, variedade de tarefas, desafios no trabalho, promoções justas, equilíbrio entre vida profissional e auto-estima. Mais uma vez, 
nesta lista, existem fatores de QVT, como a variedade de tarefas e indicadores de QVT, como equilíbrio entre vida profissional e auto-estima. Mais recentemente, Royuela, Lopez-Tamayo e Surinach (2008) identificaram 10 dimensões que descrevem QVT: valor intrínseco do trabalho, desenvolvimento de competências e habilidades para a carreira, igualdade de gênero, saúde e segurança, inclusão e acesso ao mercado de trabalho, organização do trabalho e equilíbrio trabalho-vida privada, diálogo social e envolvimento dos funcionários, diversidade e não discriminação, e desempenho global. Aqui, também há fatores como o desenvolvimento de competências e habilidades e indicadores como o valor intrínseco do trabalho.

Vale ressaltar que esse artigo trata da percepção dos docentes do Quebec e de Minas Gerais sobre a orientação frente ao trabalho e o sentido do trabalho nas universidades brasileiras e canadenses, a fim de correlacionar estas variáveis com a QVT.

\subsection{ORIENTAÇÃO PARA O TRABALHO}

Segundo Wrzesniewski (2002), a orientação para o trabalho é o modo como as pessoas vêem seu trabalho atual. Três orientações foram determinadas. A primeira é "O meu trabalho é um emprego.". As pessoas que têm este tipo de orientação não tendem a investir consideravelmente em seu trabalho ou em sua organização. Pois, eleas veem o trabalho apenas como fonte de renda e sustento. A segunda é "Meu trabalho é minha vocação.". As pessoas que têm este tipo de orientação tendem a envolver-se em seu trabalho e na organização: eles se mostram dedicadas, aplicadas, atentas ao trabalho.

O terceiro é "Meu trabalho é minha carreira.". As pessoas que têm este tipo de orientação tendem a considerar o trabalho que elas têm agora como um trampolim para outra posição. Elas vão investir o que é necessário para obter os benefícios necessários para melhorar a sua candidatura para outro cargo superior. Ou seja, para fazer uma carreira. No entanto, vale ressaltar que cada indivíduo tem essas três tendências em diferentes graus, com uma orientação dominante para a orientaçao mais valorizada pelo individuo.

A estilo de orientação para o trabalho pode ter nuancias que estão relacionadas com o sentido do trabalho ou sentido no trabalho, ou seja com as características do trabalho em si e/ou as características das relações humanas no ambiente de trabalho. Por isso, este artigo busca correlacionar estes elementos para observar seu inter-relacionamento no ambiente de trabalho acadêmico, com vistas a subsidiar gestores universitários, principalmente aqueles 
ligados a área de Gestão de Pessoas a pensar nestas variáveis ao implementar ações diversas no âmbito acadêmico.

\subsection{SENTIDO DO TRABALHO}

O sentido de trabalho está relacionado com a forma como as pessoas compreendem a sua experiência nas organizações. De acordo com Wrzesniewski, Dutton e Debebe (2003, p. 99), o sentido do trabalho pode ser definido "como o entendimento dos empregados daquilo que eles fazem no trabalho assim como a importância do que eles realmente fazem". Ambos os aspectos do sentido do trabalho estão relacionados as crenças de uma pessoa sobre a função que o trabalho desempenha na sua vida, que é afetada pelo contexto social.

Pratt e Ashforth (2003, p. 104) propõem um "modelo de distinção entre sentido do trabalho e sentido no trabalho". Neste modelo, o primeiro está relacionado com as características do trabalho e o segundo com as relações estabelecidas no trabalho. O que permite que ao indivíduo responder às seguintes perguntas: O que eu faço? Quem sou eu? e Por que estou aqui? Pratt e Ashforth (2003) apresentam a hipótese de que o sentido que o indivíduo dá ao seu trabalho e seu ambiente de trabalho está relacionada com a sua própria identidade. Vale a pena dizer que Wrzesniewski et al. (2003, p. 99) também afirmam que "os funcionários são motivados a obter um sentido para seus esforços".

Outros estudos similares buscaram analisar a relação entre o significado do trabalho e os indicadores de QVT. Nestes estudos, observou-se que existe uma relação significativa positiva entre o sentido do trabalho e o sentido no trabalho de um lado e o bem-estar psicológico, o comprometimento afetivo, comprometimento com o trabalho e o equilíbrio trabalho-vida privada de outro lado. Observou-se também que existe uma relação negativa significativa entre o sentido do trabalho e o sentido no trabalho de um lado e o sofrimento psicológico, presenteísmo, estresse relacionado ao trabalho e comprometimento de continuidade do outro (Vilas Boas \& Morin, 2013, 2013a, 2013b, 2013c). De modo complementar, Vilas Boas e Morin $(2014,2014 a)$ observaram que os fatores que descrevem as características do trabalho e das relações de trabalho estão todos positivamente correlacionadas com o sentido do trabalho para professores universitários.

\section{METODOLOGIA}


Os questionários foram enviados através Survey Monkey no primeiro semestre de 2013. O questionário foi enviado para: 625 professores da Universidade Federal de A (UFA); 378 professores da Universidade Federal de B (UFB); 995 professores da Universidade Federal de C (UFC); 1.058 professores da Universidade du Quebec à A (UQA); 414 professores da Universidade du Quebec à B (UQB), e 207 professores da Universidade du Quebec à C (UQC). Ao todo, 526 professores responderam o questionário, 106 UFA (taxa de resposta de 20,2\%), 63 UFB (taxa de resposta de 12,0\%), 105 UFC (taxa de resposta de 20,0\%), 156 de UQA (taxa de resposta de 29,7\%), 59 UQB (taxa de resposta 11,2\%) e 37 UQC (taxa de resposta de 7,0\%). As taxas de retorno foram baixas em cada universidade, por isso, é difícil realizar uma análise separada por universidade. Mas, em relação ao total de questionários em cada país, temos dados suficientes para fazer as análises comparativas.

Entre os respondentes, 248 eram mulheres e 278 eram homens. Há uma diferença significativa entre as distribuições de gênero no Brasil, em comparação com o Canadá. No Brasil, havia 106 mulheres e 178 homens, enquanto no Canadá, havia 142 mulheres e 110 homens. Assim, a amostra no Brasil tem um viés de gênero em favor dos homens.

Os professores que se voluntariaram para responder a pesquisa tinham em média 45,07 anos de idade (DP 10,481). Há uma diferença significativa na idade dos respondentes entre brasileiros e canadenses $(\mathrm{t}=-6,512, \mathrm{df}=669, \mathrm{p}<.000)$. Na verdade, entre os brasileiros $(\mathrm{N}=$ 274) a idade média é de 42,63 anos (DP 9,967) e entre os canadenses $(\mathrm{N}=252)$ a idade média é de 47,72 anos (DP 10,286). Em outras palavras, os professores brasileiros que responderam à pesquisa eram mais jovens do que os canadenses. Isso não significa, necessariamente que os professores brasileiros são, em geral, mais jovens que os canadenses, já que não temos os dados demográficos para toda a população de professores nos dois países.

Nesta amostra, $17,9 \%$ dos professores têm mestrado, $71,9 \%$ têm doutorado e $10,3 \%$ possuem pós-doutorado. Quanto ao estado civil, 15,6 \% eram solteiros, 78,4 \% eram casados ou viviam com um parceiro, 5,3\% eram separados ou divorciados e $0,4 \%$ eram viúvos. Entre os entrevistados de ambos os países, $64,1 \%$ têm filhos e a maioria deles têm duas crianças $(30,2 \%)$, em seguida uma criança $(18,8 \%)$ e 3 crianças $(12,0 \%)$. A maioria dos participantes $(99,4 \%)$ são professores de tempo integral e apenas $10,3 \%$ não possuem título de doutorado ou de $\mathrm{PhD}$. No entanto, $37,1 \%$ dos professores que participaram desta pesquisa são professores adjuntos, 26,6\% são professores associados e 19,6\% são professores titulares. Os demais respondentes $(5,9 \%)$ não informaram o seu status ou posição na instituição. 
Foi utilizada a escala de Wrzesniewski (2002) para avaliar a orientação dos professores universitários das universidades do Quebec e de Minas Gerais. Os entrevistados foram solicitados a informar em que grau eles concordaram com diversas afirmações, usando um formulário variando na escala "Concordo totalmente" a "discordo totalmente". Para avaliar o sentido do trabalho e o sentido no trabalho foi utilizado a escala de Morin (2008).

Para cada escala do questionário, a análise de componente principal, com rotação ortogonal de fatores foi realizada a fim de reduzir as variáveis observados para um número mínimo de dimensões (ou componentes) que descrevem a proporção máxima de variação para cada uma das variáveis. A estrutura fatorial das escalas foi então testada com uma análise dos eixos principais com rotação ortogonal. Foi analisada a consistência interna de cada fator, a fim de avaliar a sua confiabilidade, utilizando o alfa de Cronbach. Antes de testar as diferenças entre os dois grupos de professores, nós examinamos a consistência dos fatores, a fim de validar o modelo de QVT que pretendíamos usar utilizando o coeficiente de Pearson. A parte dessas análises, foi possível discutir a relação entre fatores de QVT nas universidades de Minas Gerais e do Quebec. A diferença de médias entre os dois grupos foi finalmente examinada usando o procedimento de teste $\mathrm{T}$ e os efeitos de tamanho dessas diferenças foram calculados quando foram identificadas diferenças significativas de médias.

\section{RESULTADOS E DISCUSSÃO}

\subsection{QUALIDADE DE VIDA NO TRABALHO}

Qualidade de Vida no Trabalho é um estado geral de bem-estar no local de trabalho. Portanto, a QVT pode ser medida por indicadores e fatores de qualidade. Se os fatores são consistentes, os índices de consistência serão fortemente correlacionados e em uma direção consistente da QVT. Os professores que perceberam uma alta qualidade de vida no trabalho na sua universidade devem obter escores elevados para a finalidade do trabalho, autonomia e desenvolvimento profissional e altos escores para as relações com colegas, retidão moral e reconhecimento. Eles devem obter pontuação positiva para a segurança no emprego. Eles também devem obter baixas contagens para cargas de trabalho. Além disso, as intercorrelações entre estes fatores devem ser significativos e em uma direção consistente, por exemplo, as oportunidades de desenvolvimento profissional e reconhecimento deve ser positivamente correlacionada e o coeficiente de Pearson deve ser significativo. 
Vilas Boas e Morin (2014a) observaram que os coeficientes de correlação são significativos e na direção esperada, mostrando a consistência das informações que essas medidas apresentam. Os fatores de QVT escolhidos, como por exemplo autonomia, oportunidades de desenvolvimento, retidão moral e carga de trabalho oferecem informações confiáveis (porque os índices de consistência interna são maiores do que 0,70 , exceto para segurança de trabalho que é 0,655 ) e informações consistentes (porque os coeficientes de Pearson são significativas e na direção esperada).

Vilas Boas e Morin (2014 e 2014a) observaram que existe uma correlação moderada positiva - entre a autonomia no trabalho e finalidade do trabalho $(0,322, \mathrm{p}<0,000)$. O tamanho do coeficiente de correlação de Pearson significa que os dois indicadores avaliam aspectos diferentes da QVT e dão informação consistente ou coerente sobre a variável latente que é suposto representar, isto é, a QVT. Da mesma forma, existe uma forte correlação positiva - entre o oportunidades de desenvolvimento profissional e a finalidade ou propósito do trabalho $(0,536, \mathrm{p}<0,000)$, mas o tamanho do coeficiente de Pearson não é suficientemente elevado para identificar um com o outro fator. Da mesma forma, há uma correlação forte - positiva - entre o desenvolvimento profissional e autonomia $(0,563, p$ $<0,000)$. Essas relações indicam claramente que eles são diferentes fatores que podem determinar as relações entre as características do trabalho.

De modo complementar há uma forte correlação - positiva - entre retidão moral e relações com os colegas $(0,716, \mathrm{p}<0,000)$, mas não alto o suficiente para confundir os dois fatores. Além disso, há uma forte correlação - positiva - entre Reconhecimento e Relações com os colegas $(0,664, \mathrm{p}<0,000)$ e Retidão moral $(0,666, \mathrm{p}<0,000)$, indicando claramente que eles são diferentes fatores que podem determinar as relações entre as próprias relações de trabalho. Existem ainda correlações fracas - positivas - entre Segurança no trabalho, Propósito, Autonomia, Desenvolvimento profissional, Retidão moral e Reconhecimento (respectivamente 0,$178 ; 0,269 ; 0,174 ; 0,150$ e $0,194, \mathrm{p}<0,000$ ). No entanto, não há correlação significativa entre o sentimento de segurança de trabalho e as cargas de trabalho dando informações consistentes sobre a percepção dos professores sobre a QVT. Essa constatação, se deve ao fato que os professores já percebem um forte sentimento de segurança, porque o trabalho neste tipo de organização é muito seguro devido à estabilidade no setor público (VILAS BOAS \& MORIN, 2014 e 2014a). 
Estas mesmas autoras observaram que também há correlações fracas - negativas entre carga física e todas as características de trabalho $(-0,130 ;-0,224 ;-0,168 ;-0,192 ;-0,200$ e $-0,226, p<0,000)$, indicando claramente que eles são fatores consistentes de determinação da QVT. Por outro lado, há uma fraca correlação - positiva - entre Carga Mental e Propósito $(0,172, \mathrm{p}<0,000)$, o que indica claramente que há duas maneiras possíveis para entender a relação entre esses fatores de QVT. No entanto, há uma correlação - negativa - um pouco fraca, mas significativa, entre Carga Mental e Desenvolvimento Profissional (-0,124, p $<0,000)$. Há também correlações fracas - negativas - entre carga emocional e todas as características de trabalho $(-0,095 ;-0,300 ;-0,204 ;-0,300 ;-0,353$ e $-0,305, \mathrm{p}<0,000)$, indicando claramente que eles são fatores consistentes na determinação da QVT. Além disso, existem correlações fracas - negativas - entre horas trabalhadas por dia e por semana, e os fatores de características do trabalho e das relações, tais como autonomia, retidão moral e reconhecimento. No entanto, existem correlações fracas - positivas - entre as horas trabalhadas e as cargas de trabalho, indicando claramente que quando as horas de trabalho diminuem as cargas de trabalho diminuem; e quando as horas de trabalho aumentam as cargas de trabalho também aumentam, como preconizado no modelo em estudo.

Em suma podemos dizer que, os professores de Minas Gerais e do Quebec sentem que têm uma boa qualidade de vida no trabalho frente aos fatores de QVT. O trabalho em si fornece-lhes as oportunidades de desenvolvimento profissional, reconhecimento e desenvolvimento de boas relações com os colegas. Eles podem ver a utilidade social do seu trabalho (propósito de trabalho) e percebem altos escores de autonomia. Além disso, a retidão moral é bem avaliada pelos professores de Minas Gerais, bem como do Quebec. À primeira vista, parece que este é um ambiente de trabalho de alta qualidade.

\subsection{ORIENTAÇÃO PARA O TRABALHO}

A orientação para o trabalho procura descrever o foco de atenção dos trabalhadores frente ao seu trabalho. No geral eles podem adotar três tendências ou posturas: ver o trabalho como um emprego, como uma carreira ou como uma vocação. Dependendo do olhar do profissional ele pode se identificar mais ou menos com seu trabalho. Cada uma das três tendências apresenta diferentes graus, com uma orientação dominante. No geral, a Tabela 1 mostra que a orientação dominante para professores universitários em Quebec e Minas Gerais 
é a vocação, seguida da carreira. Neste ambiente acadêmico pode-se perceber que os poucos professores vêem o trabalho apenas como um emprego.

Tabela 1 Estatística descritiva dos tipos de orientação para o trabalho

\begin{tabular}{|l|l|l|l|l|l|}
\hline & N & Minimo & Maximo & Média & $\begin{array}{l}\text { Desvio- } \\
\text { padrão }\end{array}$ \\
\hline Meu trabalho é minha vocação & 520 & 12,00 & 60,00 & 45,6231 & 8,24272 \\
\hline Meu trabalho é um emprego & 520 & 10,00 & 56,67 & 21,2628 & 9,35785 \\
\hline Meu trabalho é minha carreira & 520 & 10,00 & 60,00 & 28,7019 & 13,17211 \\
\hline N valido (listwise) & 520 & & & & \\
\hline
\end{tabular}

Fonte: Dados da pesquisa

Com relação a orientação para o trabalho entre os dois grupos de professores, pode-se observar que todos as médias são mais elevadas para os professores mineiros. O maior desvio é observado na direção de carreira (Tabela 2). Para saber se essas diferenças são significativas foi realizado o teste t. No geral, estes resultados coorboram com os estudos sobre a orientação do trabalho realizados por Wrzesniewski, Dutton \& Debebe (2003).

Tabela 2 Estatística descritiva da orientação para o trabalho de professores de Minas Gerais e do Quebec

\begin{tabular}{|l|c|c|c|c|c|}
\hline Tipo de orientação & Região & N & Média & $\begin{array}{c}\text { Desvio } \\
\text { Padrão }\end{array}$ & $\begin{array}{c}\text { Erro padrãa } \\
\text { da média }\end{array}$ \\
\hline $\begin{array}{l}\text { Meu trabalho é uma } \\
\text { vocação }\end{array}$ & Minas Gerais & 268 & $\mathbf{4 6 , 2 9 8 5}$ & 7,55904 &, 46174 \\
\cline { 2 - 6 } Meu trabalho é um & Quebec & 252 & 44,9048 & 8,87131 &, 55884 \\
\hline $\begin{array}{l}\text { emprego } \\
\text { Meu trabalho é uma } \\
\text { carreira }\end{array}$ & Minas Gerais & 268 & $\mathbf{2 3 , 3 8 3 1}$ & 9,72348 &, 59396 \\
\cline { 2 - 6 } & Quebec & 252 & 19,0079 & 8,40192 &, 52927 \\
\cline { 2 - 6 } & Quebec & 252 & 25,0595 & 12,65133 &, 77868 \\
\hline
\end{tabular}

Fonte: Dados da pesquisa

De acordo com os testes $t$ da Tabela 3, a média das diferenças são significativas para a orientação para o emprego e orientaçao para a carreira. Mas, é importante notar que a orientação para a vocação é quase estatisticamente significativa. O tamanho do efeito dessas diferenças pode ser medido através da diferença de médias entre os dois grupos, com o cálculo do índice eta quadrado $(\eta 2)$. O tamanho do efeito para a orientação meu trabalho é um emprego é pequeno $(0,01)$ e para a orientação, meu trabalho é uma carreira também é pequeno $(0,01)$.

Considerando-se os resultados para esses dois grupos, pode-se concluir que a orientação do trabalho como um emprego e como uma carreira seria mais forte para os 


\section{SENTIDO DO TRABALHO E ORIENTAÇÃO PARA O TRABALHO: UM ESTUDO EM UNIVERSIDADES \\ PÚBLICAS DE MINAS GERAIS E DO QUEBEC \\ DOI: http://dx.doi.org/10.5007/1983-4535.2015v8n4p117}

professores de Minas Gerais que a orientação do trabalho como um emprego e como uma carreira para os professores do Quebec.

Tabela 3 Diferença de médias entre 268 professores do Quebec e 252 professores mineiros

\begin{tabular}{|c|c|c|c|c|c|c|}
\hline $\begin{array}{ll}\text { Tipo } & \text { de } \\
\text { orientação }\end{array}$ & Região & $\begin{array}{l}X \\
\text { Média }\end{array}$ & $\begin{array}{l}\text { S } \\
\text { Desvio } \\
\text { padrão } \\
\end{array}$ & $\mathbf{T}$ & $\begin{array}{l}\text { Dll } \\
\text { Graus de } \\
\text { liberdade }\end{array}$ & $\begin{array}{l}\text { Sig } \\
\text { (bi) }\end{array}$ \\
\hline \multirow[t]{2}{*}{$\begin{array}{l}\text { Meu trabalho é } \\
\text { uma vocação }\end{array}$} & $\begin{array}{l}\text { Minas } \\
\text { Gerais }\end{array}$ & 46,2985 & ,72138 & 1,932 & 518 &, 054 \\
\hline & Quebed & 44,9048 & ,72492 & & & \\
\hline \multirow[t]{2}{*}{$\begin{array}{l}\text { Meu trabalho é } \\
\text { um emprego }\end{array}$} & $\begin{array}{l}\text { Minas } \\
\text { Gerais }\end{array}$ & 23,3831 & ,79912 & 5,499 & 514,375 & ,000 \\
\hline & Quebec & 19,0079 & ,79556 & & & \\
\hline \multirow[t]{2}{*}{$\begin{array}{l}\text { Meu trabalho é } \\
\text { uma carreira }\end{array}$} & $\begin{array}{l}\text { Minas } \\
\text { Gerais }\end{array}$ & 32,1269 & 1,11448 & 6,343 & 516,488 & ,000 \\
\hline & Quebec & 25,0595 & 1,11422 & & & \\
\hline
\end{tabular}

\subsection{SENTIDO DO TRABALHO E ORIENTAÇÃO PARA O TRABALHO}

A fim de entender melhor a relação entre as a orientação para o trabalho e o sentido do trabalho (características do trabalho) e o sentido no trabalho (características das relações de trabalho), nós calculamos as correlações entre estas variáveis. A Tabela 4 mostra que existe correlação positiva moderada, quase forte $(0,498)$, entre o sentido do trabalho e orientação do trabalho para a vocação. Ou seja, quando o ambiente de trabalho é adequado, a tendência do profissional ver seu trabalho como vocação aumenta. Na mesma tabela podemos observar que existe correlação moderada significativa e positiva $(0,402)$ também entre o sentido no trabalho e a vocação. O que indica que se as relações de trabalho são favoráveis os professores universitários tendem a ver o trabalho que eles escolheram como uma vocação.

\subsection{DISCUSSÃO}

A pesquisa demandou que professores de Minas Gerias e do Quebec avaliassem a sua QVT, para posteriormente comparar as percepções destes profissionais com a orientação para o trabalho. Em geral, os dois grupos parecem apreciar a qualidade de vida no trabalho nas suas respectivas universidades. No entanto, há uma pequena diferença entre a avaliação que foi feita pelos professores de Minas Gerais e do Quebec. Na verdade, parece que os professores do Quebec encontram mais qualidade de vida no trabalho do que os de Minas 
Gerais em relação aos fatores de qualidade de vida estudados. Os resultados desta pesquisa mostraram claramente que esses professores fazem a diferença entre os fatores de QVT, assim como observado por Morin (2003, 2008) e Morin e Dassa (2004) em outros contextos pesquisados.

Tabela 4 Coeficientes de correlação de Pearson entre o significado do trabalho e orientação para o trabalho

\begin{tabular}{|l|c|c|c|c|}
\hline & & Vocação & Emprego & Carreira \\
\hline Sentido do trabalho & $\mathbf{r}$ & $\mathbf{, 4 9 8}$ &, 017 &, 015 \\
\cline { 2 - 5 } & Sig (bil) &, 000 &, 692 &, 735 \\
\cline { 2 - 5 } & $\mathrm{N}$ & 520 & 520 & 520 \\
\hline Sentido no trabalho & $\mathbf{R}$ & $\mathbf{4 0 2}$ &,- 032 &, 035 \\
\cline { 2 - 5 } & Sig (Bil) &, 000 &, 461 &, 432 \\
\cline { 2 - 5 } & $\mathrm{n}$ & 520 & 520 & 520 \\
\hline
\end{tabular}

Fonte: Dados da pesquisa

Os professores do Quebec têm mais autonomia no trabalho e mais oportunidades para o desenvolvimento profissional do que os professores de Minas Gerais. Os professores do Quebec tendem a encontrar mais retidão moral e reconhecimento do que os professores mineiros. Eles também são capazes de controlar melhor suas relações emocionais no local de trabalho do que os seus colegas mineiros. Mas, os professores do Quebec apontaram ter uma carga física maior de trabalho que os professores mineiros. Por outro lado, os professores de Minas Gerais tendem a sentir mais carga mental e trabalham mais horas por dia do que os colegas do Quebec.

Por outro lado, existe uma relação negativa entre os fatores relacionados ao trabalho características de organização e as cargas de trabalho. Vale a pena lembrar que a carga emocional do trabalho foi negativamente correlacionada com a autonomia. De fato, a carga de trabalho é um fator de risco psicossocial bem confirmado e atualmente a carga de trabalho dos professores universitários é muito alta devida as crescentes exigências de pesquisa e publicação, como observado por Catano et al. (2010), Miller, Taylor e Bedeian (2011), Winefield et al. (2003), Vannini (2006) e Gillespie et al. (2001). Diante do exposto, corroboramos o exposto por Wortman, Biernat e Lang (1991) de que os profissionais e as organizações devem buscar estratégias de enfrentamento do excesso de carga de trabalho, o que a nosso ver vai minimizar os efeitos negativos no sentido do trabalho.

\section{CONSIDERAÇÕES FINAIS}


Esta pesquisa foi concebida para avaliar a qualidade de vida no trabalho dos professores de universidades públicas e comparar as avaliações dos professores que trabalham no estado de Minas Gerais, com a QVT de professores que trabalham no Quebec. Além disso, buscou-se relacionar esses fatores com os indicadores orientação para o trabalho e indicadores de sentido/significado do trabalho. Neste estudo exploratório, os resultados mostram que, em geral, os professores sentem que têm uma boa qualidade de vida no trabalho. Professores do Quebec encontram mais autonomia no trabalho e eles têm mais oportunidades de desenvolvimento profissional do que os brasileiros. Eles também percebem mais retidão moral e reconhecimento no seu trabalho do que os colegas brasileiros. No entanto, os professores universitários de Quebec sentem que têm uma carga física maior do que os professores de Minas Gerais. Por outro lado, os professores mineiros apontaram mais carga emocional na realização de seu trabalho do que os seus colegas do Quebec e eles trabalham mais horas por dia também. Observou-se também que a orientação para o trabalho está positivamente correlacionada com o sentido do trabalho e com o sentido no trabalho e que a orientação para o trabalho como vocação apresentou correlação significativa positiva nesta amostra de professores universitários.

Em resumo, as diferenças entre os dois grupos são pequenos e os seus efeitos de tamanho também são pequenos. As diferenças significativas são em dois aspectos: o trabalho visto como emprego e o trabalho visto como carreira que é maior para os professores de Minas Gerais. Este estudo mostrou também que o significado do trabalho está diretamente correlacionado com a vocação pela profisssão na academia.

Promover a qualidade de vida no trabalho é um desafio para o setor de gestão universitária, mas este tema deve ser abordado em novos estudos pois toda a sociedade vai colher os benefícios. Tanto em termos de desempenho profissional e da qualidade dos serviços oferecidos à sociedade, bem como, em termos de vida pessoal e profissional. No entanto, vale lembrar que a orientação para o trabalho pode ser um bom indicador para promover adaptações junto aos professores. Pois, os estudos sobre o sentido no trabalho mostram que a identificação com o trabalho em si e a aproximação com os colegas e superiores é fundamental para encontrarmos um trabalho que nos dê prazer. O que pode contribuir com a melhoria da Qualidade de Vida no Trabalho.

Para novos estudos sobre sentido do trabalho e orientação para o trabalho nós sugerimos a realização de pesquisas similares em outros estados do Brasil e em outras regiões 
do Canadá. Sugerimos também a realização de análises comparativas entre gênero e mesmo entre diferentes faculdades ou institutos de outras universidades públicas ou privadas. $\mathrm{Na}$ prática sugerimos enfatizar a realização de pesquisas e diagnósticos similares pelas próreitoras de Gestão de Pessoas de modo a traçar planos e programas que melhorem as condições de trabalho e as relações de trabalho para que os professores universitários continuem vendo a carreira universitária como um vocação e como um ideal que contribui com o progresso da ciência e com o desenvolvimento da cidadania.

\section{Agradecimento}

Os autores agradecem à CAPES pelo financiamento desta pesquisa realizada na HEC Montreal.

\section{REFERÊNCIAS}

Boisvert, M. P. (1977). The quality of working life: an analysis. Human Relations, 30(2), 155160.

Catano, V.; Francis, L.; Haines, T.; Kirpalani, H.; Shannon, H.; Stringer, B. \& Lozanzki, L. (2010). Occupational Stress in Canadian Universities: a national survey. International Journal of Stress Management. 17(3), 232-258.

Davis, L. E. \& Cherns, A. B. (1975). (Eds). The Quality of Working Life. (Vol 1. Problems, Prospects, and the State of the Art), New York, USA, Free Press.

Gillespie, N. A.; Walsh, M.; Winefields, A. H.; Dua, J. \& Stough C. (2001). Occupational Stress in Universities: staff perceptions of the causes, consequences and moderators of stress. Work \& Stress. 15(1), 53-72.

Hancock, P. A. \& Verwey, W. B. (1997). Fatigue, workload and adaptive driver systems. Accident Analysis and Prevention. 29, 495-506.

Hancock, P. A.; Rodenberg, G. J.; Mathews, W. \& Vercruyssen, M. (1988). Estimation of duration and mental workload at different times of the day by males and females. Proc Human Factors. 32, 857-861.

Ketchum, L. D. \& Trist, E. (1992). All teams are not created equal: how employee empowerment really works. Newbury Park: Sage.

Kinman. G. \& Jones, F. (2008). A Life Beyond Work? Job Demands, Work-Life Balance, and Wellbeing in UK Academics. Journal of Human Behavior in the Social Environment, 17(1/2), 41-60. doi: 10.1080/10911350802165478 
Levine, M. F. (1983). Self-developed QWL measures. Journal of Occupational Behavior, $4(1), 35-46$.

Miller, A. N. Taylor, S. G. \& Bedeian, A. G. (2011). Publish or Perish: Academic life as management faculty live it. The Career Development International. 16(5), 422-425. Doi: $10.1108 / 13620431111167751$

Morin, E. M. (1997). Le sens du travail pour des gestionnaires francophones. Psychologie du travail et des organisations, 2 (3), 26-45.

Morin, E. M. (2001). Os sentidos do trabalho. Revista de Administração de Empresas, 41(3), 8-19.

Morin E. M. (2003). Sens du travail. Définition, mesure et validation. In C. Vandenberghe, N. Delobbe et G. Karnas. (éds), Dimensions individuelles et sociales de l'investissement professionnel. Actes du XIIe Congrès de psychologie du travail et des organisations (2, pp. 11-20). Louvain-la-Neuve, UCL Presses universitaires de Louvain.

Morin, E. M. (2008). Sens du travail, santé mentale au travail et engagement organisationnel, Études et recherches, (Rapport R-543 - avec la collaboration de F. Aranha, FGV-EASP), Montreal, QC, Canada, IRSST.

Morin E. M. \& Cherré B. (1999). Les cadres face au sens du travail. Revue Française de Gestion, 126, 83-93.

Morin, E. M. \& Dassa, C. (2004). Characteristics of a meaningful work: Construction and validation of a scale. HEC Montréal/Université de Montréal, Montréal, QC, Canada.

Royuela, V., Lopez-Tamayo, J. \& Surinach, J. (2008). The institutional vs. the academic definition of the quality of work life. What is the focus of the European Commission? Social Indicators Research. 86, 401-415.

Van Vuuren, C. V., \& Klandermans, P. G. (1990). Individual reactions to job insecurity: An integrated model. In P. J. D. Drenth, J. A. Sergeant, \& R. J. Takens (Eds.), European perspectives in psychology (3, pp. 133-146). Chichester, England: Wiley.

Vannini, P. (2006). Dead Poets' Society: Teaching, Publish-or-Perish and Professors' Experience of Authenticity. Symbolic Interaction. 29(2), 235-257. Doi:

10.1525/si.2006.29.2.235

Vilas Boas, A. A. \& Morin, E. M. (2013a, July). El sentido del trabajo y la calidad de vida en el trabajo dentro de los establecimientos públicos de enseñanza superior: una comparación entre Brasil y Canadá. Boletin Informativo, Ciudad de Quatemala: Ministerio de Relaciones Exteriores - El Heraldo Diplomático - Biblioteca Mario Monteforte Toledo, 56.

Vilas Boas, A. A. \& Morin, E. M. (2013b, September). Indicadores de qualidade de vida no trabalho para instituições públicas de ensino superior: uma comparação entre Brasil e Canadá. 
Anais do Encontro Nacional da Associação Nacional de Pós-graduação e Pesquisa em Administração, Rio de Janeiro, RJ, Brazil, 37.

Vilas Boas, A. A. \& Morin, E. M. (2013, May). Le sens du travail et la qualité de vie au travail dans les établissements publics d'enseignement supérieur: une comparaison entre le Brésil et le Canada. Poursuites de la Colloque International AFIRSE: Recherche en éducation : Continuité, rupture ou limites? UQAM, Montréal, QC, Canada.

Vilas Boas, A. A. \& Morin, E. M. (2013c, September). Quality of Working Life in Public Higher Education Institutions: the perception of Brazilian and Canadian professors. International Journal of Business and Social Science. 4(12, Special Issue), 67-77.

Vilas Boas, A. A. \& Morin, E. M. (2014). Fatores de Qualidade de Vida no Trabalho em Universidades Públicas: Uma Comparação entre Brasil e Canadá. Anais do $14^{\circ}$ Congresso de Stress da ISMA (Internacional Stress Management Asssociation) e do $16^{\circ}$ Fórum de Qualidade de Vida no Trabalho, Porto Alegre, RG, Brasil.

Vilas Boas, A. A. \& Morin, E. M. (2014). Sentido do Trabalho e Fatores de Qualidade de Vida no Trabalho para Professores de Universidades Públicas do Brasil e do Canadá. Anais do Encontro Nacional da Associação Nacional de Pós-graduação e Pesquisa em Administração, Rio de Janeiro, RJ, Brazil, 38.

Winefield, A.H., Gillespie. N., Slough, C. Dua. J., Hapuarachchi, J., \& Boyd, C. (2003). Occupational stress in Australian University Staff. International Journal of Stress Management. 10, 51-63.

Wortman, C, Biernat, M. \& Lang. E. (1991). Coping with role overload. In M. Frankenhaeuser. U. Lundberg \& M. Chesney (Eds.) Women. Work and Health: Stress and Opportunities, London: Plenum.

Wrzesniewski, A., Dutton, J. E. \& Debebe, G. (2003). Interpersonal Sensemaking and the Meaning of Work. Research in Organizational Behavior, Vol 25, 93-135. ISSN: 01913085/doi:10.1016/ 